Angew Chem Int Ed Engl. 2018 October 26; 57(44): 14529-14532. doi:10.1002/anie.201806015.

\title{
Nickel-Catalyzed Enantioconvergent Borylation of Racemic Secondary Benzylic Electrophiles**
}

\author{
Zhaobin Wang, Shoshana Bachman, Alexander S. Dudnik, and Prof. Gregory C. Fu* \\ Division of Chemistry and Chemical Engineering, California Institute of Technology, Pasadena, \\ CA, 91125 (USA)
}

\section{Abstract \\ Nickel-catalyzed cross-coupling has emerged as the most versatile approach to date for achieving enantioconvergent carbon-carbon bond formation using racemic alkyl halides as electrophiles. In contrast, there have not yet been reports of the application of chiral nickel catalysts to corresponding reactions with heteroatom nucleophiles to produce carbon-heteroatom bonds with good enantioselectivity. Herein, we establish that a chiral nickel/pybox catalyst can borylate racemic secondary benzylic chlorides to provide enantioenriched benzylic boronic esters, a highly useful family of compounds in organic synthesis. The method displays good functional group compatibility (e.g., unimpeded by the presence of an indole, a ketone, a tertiary amine, or an unactivated alkyl bromide), and both of the catalyst components $\left(\mathrm{NiCl}_{2} \cdot\right.$ glyme and a pybox ligand) are commercially available.}

\section{Graphical Abstract}

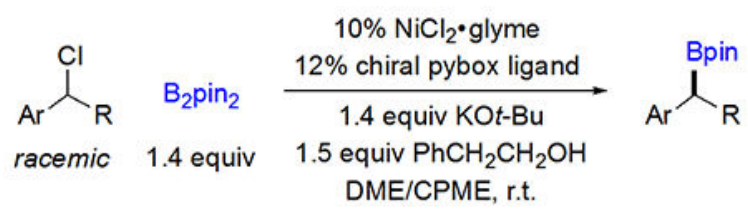

\begin{abstract}
Nickel-catalyzed stereoconvergent cross-couplings of racemic alkyl electrophiles have been expanded to include C-heteroatom bond construction for the first time, specifically, a chiral nickel/pybox catalyst has been shown to borylate benzylic chlorides to produce enantioenriched benzylic boronic esters, a useful family of compounds in organic synthesis.
\end{abstract}

\section{Keywords}

asymmetric catalysis; boron; cross-coupling; nickel

\footnotetext{
** Support has been provided by the National Institutes of Health (National Institute of General Medical Sciences, grant R01GM062871). We thank Dr. Scott C. Virgil, Dr. David G. VanderVelde, Dr. Xin Mu, and Dr. Yufan Liang for assistance and helpful discussions.

*gcfu@caltech.edu.

Supporting information for this article is given via a link at the end of the document.
} 
Enantioenriched benzylic boronic esters are an important family of target compounds in organic synthesis. ${ }^{[1,2,3,4]}$ From a practical perspective, boronic esters are attractive compared with organometallic reagents such as Grignard reagents and trialkylboranes due to their comparatively high air- and moisture-stability. ${ }^{[5]}$ Furthermore, the ability to transform the $\mathrm{C}-\mathrm{B}$ bond of boronic esters, with stereochemical fidelity, into bonds such as $\mathrm{C}-\mathrm{C}, \mathrm{C}-\mathrm{N}, \mathrm{C}-$ $\mathrm{O}$, and $\mathrm{C}-$ halogen render them highly versatile intermediates in organic chemistry. ${ }^{[6]}$ Due to these appealing features, an array of methods have been developed for the synthesis of enantioenriched benzylic boronic esters, ${ }^{[1]}$ including catalytic asymmetric processes such as the hydroboration of alkenes ${ }^{[2]}$ and the hydrogenation of alkenylboronic esters. ${ }^{[3,4]}$

We and others have recently described a variety of nickel-catalyzed enantioconvergent crosscouplings of nucleophiles with racemic alkyl electrophiles; to date, progress in this area has been limited to the use of carbon-based nucleophiles to form $\mathrm{C}-\mathrm{C}$ bonds (Figure 1a). ${ }^{[7]}$ Clearly, expanding the scope to include $\mathrm{C}$-heteroatom bond construction would substantially enhance the usefulness of this strategy in synthesis. Aware of the importance of enantioenriched organoboron compounds, we chose to explore the possibility that a boron reagent might serve as the first effective heteroatom-based nucleophile in nickel-catalyzed enantioconvergent cross-couplings. ${ }^{[8,9,10,11]}$ Herein, we establish the viability of this approach (Figure 1b), specifically, we report a nickel/pybox-catalyzed borylation that provides enantioenriched boronic esters in good enantiomeric excess from an array of racemic secondary benzylic chlorides [Eq. (1)].

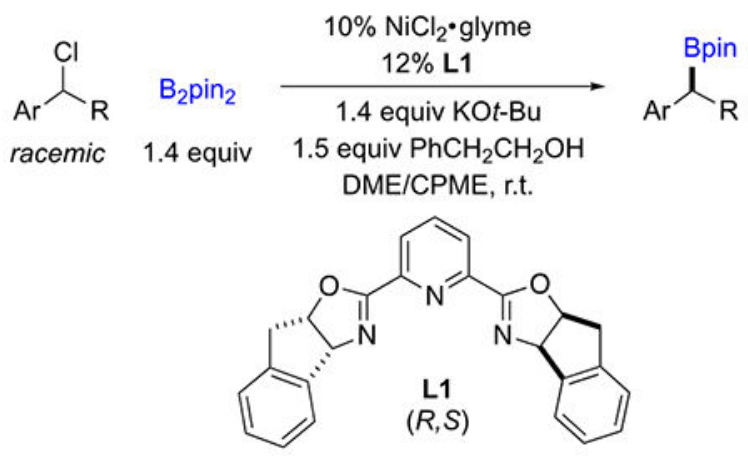

Upon exploring a range of conditions, we determined that the enantioconvergent borylation of the racemic secondary benzylic chloride by $\mathrm{B}_{2} \mathrm{pin}_{2}$ that is illustrated in Table 1 can be achieved in $87 \%$ yield and $85 \%$ ee in the presence of a chiral nickel/pybox catalyst (entry 1); $\mathrm{NiCl}_{2} \cdot$ glyme, the pybox ligand $\mathbf{L 1}$, and $\mathrm{B}_{2} \mathrm{pin}_{2}$ are all commercially available. Essentially no $\mathrm{C}-\mathrm{B}$ bond formation is detected if $\mathrm{NiCl}_{2} \bullet$ glyme is omitted (entry 2), whereas a small amount is observed if pybox ligand $\mathbf{L} \mathbf{1}$ or the primary alcohol is absent (entries 3 and 4). ${ }^{[12]}$ The use of pybox ligands $\mathbf{L} \mathbf{2}$ and $\mathbf{L 3}$, rather than $\mathbf{L 1}$, leads to excellent yield but low enantioselectivity (entries 5 and 6), whereas bis(oxazoline) ligand $\mathbf{L} \mathbf{4}$ provides poor yield and ee (entry 7). Lowering the temperature does not result in an enhancement in enantioselectivity (entry 8 ), and the use of a single solvent leads to a loss in yield with no change in ee (entries 9 and 10). Decreasing the catalyst loading $(10 \% \rightarrow 5 \%)$ or the amount 
of nucleophile (1.4 equiv $\rightarrow 1.1$ equiv) has only a minor impact on the course of the reaction (entries 11 and 12). The enantioconvergent borylation is not highly water- or air-sensitive: even if it is conducted in the presence of a full equivalent of water or in a capped vial under air, a substantial amount of product is formed, in fairly good ee (entries 13 and 14).

Next, we explored the scope of this new method for the catalytic asymmetric synthesis of enantioenriched benzylic boronic esters (Table 2). We have determined that the alkyl group (R) of the electrophile can vary in steric demand from Me to Cy (entries 1-5), although the presence of a bulky cyclohexyl substituent leads to somewhat lower yield and ee (entry 5). Functional groups such as an ether, an ester, and an unactivated primary alkyl chloride are compatible with the borylation conditions (entries 7-9). With regard to the aryl substituent, it may be substituted in the para, meta, or ortho position (entries 10-14), and it can be a 1naphthyl group (entry 15); however, if it is a 6-dibenzofuryl substituent, the reaction proceeds in good yield but moderate ee (entry 16). The method can be applied not only to an acyclic, but also to a cyclic, benzylic chloride (entry 17). On a gram-scale (1.42 g of product), the borylation illustrated in entry 3 proceeds in $85 \%$ yield and $88 \%$ ee.

By conducting an enantioconvergent borylation in the presence of a variety of additives, we have further examined the functional-group tolerance of the method (Figure 2). We have determined that an aryl chloride, aryl tosylate, epoxide, unactivated secondary alkyl bromide, tertiary amide, ketone, tertiary amine, benzofuran, and indole have little or no impact on $\mathrm{C}-\mathrm{B}$ bond formation, and the additive can be recovered virtually quantitatively at the end of the reaction $(>90 \%)$. On the other hand, the presence of a thioether, aldehyde, or nitroalkane impedes the borylation.

As mentioned at the outset, enantioenriched benzylic boronic esters are versatile intermediates in organic synthesis that can be converted with high stereochemical fidelity into other useful families of compounds; several representative examples are provided in Figure 3. Thus, the illustrated boronic ester can be aminated, hydroxymethylated, and arylated (a variety of bioactive compounds include tertiary stereocenters that bear two different aryl substituents ${ }^{[13]}$ ) without erosion in enantiomeric excess. ${ }^{[6]}$

We have determined that, with a given enantiomer of catalyst, nickel-catalyzed borylation proceeds with essentially identical yield and enantioselectivity, regardless of the original configuration of the electrophile, establishing that the stereochemistry of the borylation is fully controlled by the chiral catalyst [Eq. (2) and (3)]. Furthermore, at partial conversion, the ee of the electrophile is unchanged, indicating that the chiral catalyst reacts with the two enantiomers of the electrophile at essentially identical rates, that the electrophile does not undergo racemization under the reaction conditions, and that $\mathrm{C}-\mathrm{Cl}$ cleavage by the catalyst is irreversible. ${ }^{[14]}$ 

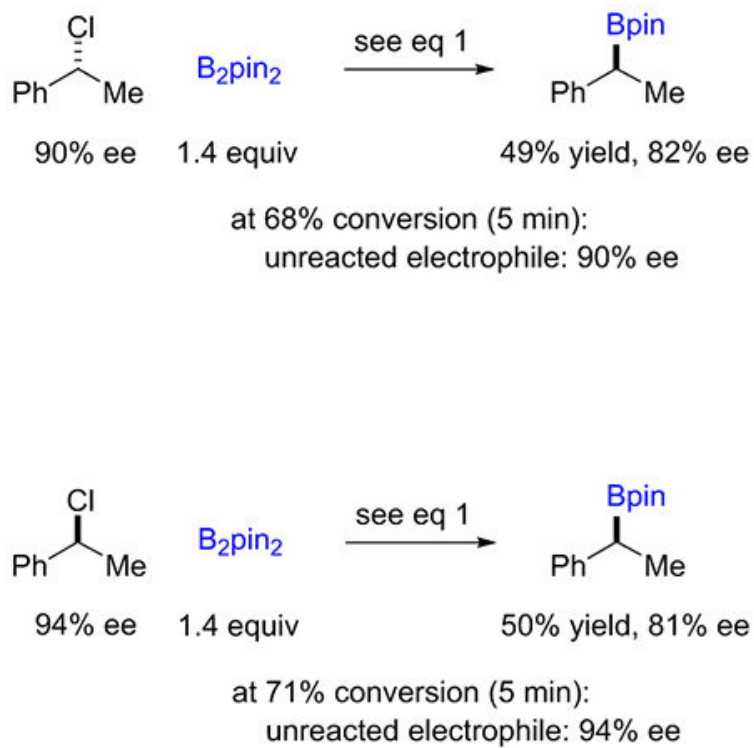

In summary, we have enlarged the scope of nickel-catalyzed stereoconvergent crosscouplings of alkyl electrophiles to include $\mathrm{C}$-heteroatom bond construction for the first time, specifically, transforming racemic benzylic chlorides into enantioenriched benzylic boronic esters, a useful family of compounds in organic synthesis. The method displays good functional-group compatibility, and both of the catalyst components $\left(\mathrm{NiCl}_{2} \cdot\right.$ glyme and pybox ligand L1) are commercially available. Investigations of other C-heteroatom bondforming processes, including enantioconvergent reactions, as well as mechanistic studies, are underway.

\section{Supplementary Material}

Refer to Web version on PubMed Central for supplementary material.

\section{References}

[1]. For an overview with leading references, see:Collins BSL, Wilson CM, Myers EL, Aggarwal VK, Angew. Chem. Int. Ed 2017, 56, 11700-11733.

[2]. a) Hayashi T, Matsumoto Y, Ito Y, J. Am. Chem. Soc 1989, 111, 3426-3428.b) Brown JM, Nguyen BN, Science of Synthesis, Stereoselective Synthesis 2011, 1, 295-324.

[3]. Ueda M, Saitoh A, Miyaura N, J. Organomet. Chem 2002, 642, 145-147.

[4]. For representative recent reports of methods for the synthesis of generic (i.e., no extraneous directing/functional group) enantioenriched benzylic boronic esters, see:a) Lovinger GJ, Morken JP, J. Am. Chem. Soc 2017, 139, 17293-17296. [PubMed: 29116784] b) Guo J, Cheng B, Shen X, Lu Z, J. Am. Chem. Soc 2017, 139, 15316-15319. [PubMed: 29045129] c) Sun C, Potter B, Morken JP, J. Am. Chem. Soc 2014, 136, 6534-6537. [PubMed: 24564423]

[5]. Boronic Acids: Preparation and Applications in Organic Synthesis, Medicine and Materials, Hall DG, Ed., Wiley-VCH, Weinheim, 2011.

[6]. For representative examples and leading references, see:a) Leonori D, Aggarwal VK, Angew. Chem. Int. Ed 2015, 54, 1082-1096.b) Mlynarski SN, Karns AS, Morken JP, J. Am. Chem. Soc 
2012, 134, 16449-16451. [PubMed: 23002712] c) Chen A, Ren L, Crudden CM, J. Org. Chem 1999, 64, 9704-9710.d) Llaveria J, Leonori D, Aggarwal VK, J. Am. Chem. Soc 2015, 137, 10958-10961. [PubMed: 26292943] e) Bonet A, Odachowski M, Leonori D, Essafi S, Aggarwal VK, Nat. Chem 2014, 6, 584-589. [PubMed: 24950327] f) Larouche-Gauthier R, Elford TG, Aggarwal VK, J. Am. Chem. Soc 2011, 133, 16794-16797. [PubMed: 21939203]

[7]. For reviews, see:a) Fu GC, ACS Cent. Sci 2017, 3, 692-700. [PubMed: 28776010] b) Iwasaki T, Kambe N, Top. Curr. Chem 2017, 374, 1-36.

[8]. To the best of our knowledge, the only transition-metal-catalyzed enantioconvergent borylation of an alkyl electrophile that has been reported to date is a copper-catalyzed coupling of cyclic allylic ethers/carbonates:Ito H, Kunii S, Sawamura M, Nat. Chem 2010, 2, 972-976. [PubMed: 20966955]

[9]. For stereospecific nickel-catalyzed borylations of enantioenriched electrophiles, see:Basch CH, Cobb KM, Watson MP, Org. Lett 2016, 18, 136-139. [PubMed: 26679212]

[10]. For early examples of metal-catalyzed, non-enantioselective borylations of primary benzylic halides, see:Giroux A, Tetrahedron Lett 2003, 44, 233-235.

[11]. For a review of metal-catalyzed borylations of aryl and alkenyl electrophiles, see:Ishiyama T, Miyaura N, Boronic Acids; Hall DG, Ed.; Wiley-VCH, Weinheim, 2011, pp 135-169.

[12]. We have established that, in the presence of the diboron reagent $\left({ }^{11} \mathrm{~B}\right.$ NMR: $\left.\delta 30 \mathrm{vs} . \mathrm{BF}_{3} \cdot \mathrm{Et}_{2} \mathrm{O}\right)$, $\mathrm{KO} t$ - Bu, and $\mathrm{PhCH}_{2} \mathrm{CH}_{2} \mathrm{OH}$, a boron-ate complex is formed $\left({ }^{11} \mathrm{~B} \mathrm{NMR}: \delta 3\right)$, which likely serves as the nucleophile in the transmetalation step of the catalytic cycle, generating a nickelboryl intermediate.

[13]. For example:Zoloft ${ }^{\mathrm{TM}}$ (Khouzam HR, Ernes R, Gill T, Roy R, Compr. Ther 2003, 29, 47-53)

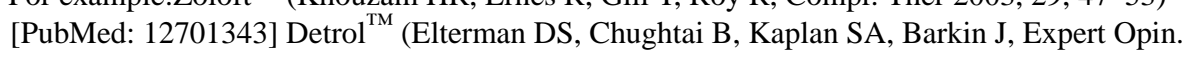
Pharmacother 2013, 14, 1987-1991). [PubMed: 23885788]

[14]. a) For an example of (modest) kinetic resolution in a nickel-catalyzed enantioconvergent crosscoupling of a racemic alkyl electrophile, see: Lundin PM, Fu GC, J. Am. Chem. Soc 2010, 132, 11027-11029. [PubMed: 20698665] b) For an example of stereoisomerization of an alkyl electrophile in a nickel-catalyzed stereoconvergent cross-coupling, see: Mu X, Shibata Y, Makida Y, Fu GC, Angew. Chem. Int. Ed 2017, 56, 5821-5824. 


\section{Nickel-catalyzed enantioconvergent cross-couplings:}

a) Previous work: Limited to $C-C$ bond formation<smiles>[R][M]OC([R])[R]</smiles>

racemic

b) This study: $C-B$ bond formation<smiles>CC([Hg])C(C)(C)C</smiles>

racemic
$\mathrm{B}_{2} \operatorname{pin}_{2}$

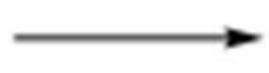

Figure 1.

Nickel-catalyzed enantioconvergent cross-couplings of alkyl electrophiles. 
$\overbrace{\mathrm{Ph}}^{\mathrm{Cl}}$

racemic
$\mathrm{B}_{2} \mathrm{pin}_{2}$

1.4 equiv

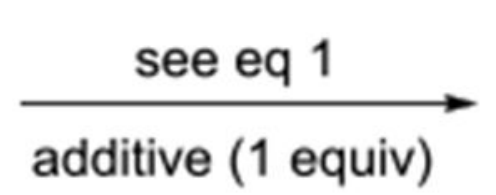

Bpin<smiles>CCC(C)P</smiles>

no additive:

$87 \%$ yield, $85 \%$ ee

\section{Borylation product: $>80 \%$ yield, $\geq 83 \%$ ee Recovery of additive: $\geq 90 \%$}<smiles>Clc1ccccc1</smiles><smiles>[O-]c1ccccc1</smiles><smiles>FC(F)(F)c1ccccc1</smiles><smiles>C1CC2CC3OC3C2C1</smiles><smiles>BrC1CCCCC1</smiles><smiles>CN(C)C(=O)c1ccccc1</smiles><smiles>CC(=O)c1ccccc1</smiles>

$\mathrm{NEtCy}_{2}$
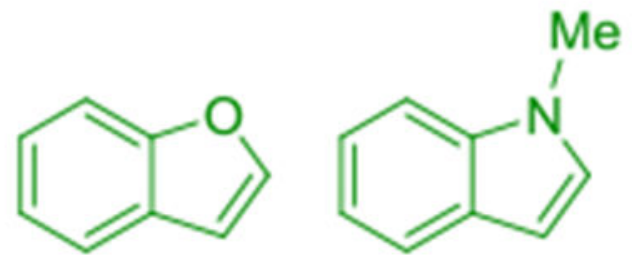

Yield of product: $<40 \%$<smiles>CCSc1ccccc1</smiles><smiles>O=CC1CCCCC1</smiles><smiles>O=[N+]([O-])C1CCCCC1</smiles>

Figure 2.

Enantioconvergent borylation of a racemic benzylic chloride: functional-group compatibility. Each additive was examined individually. 

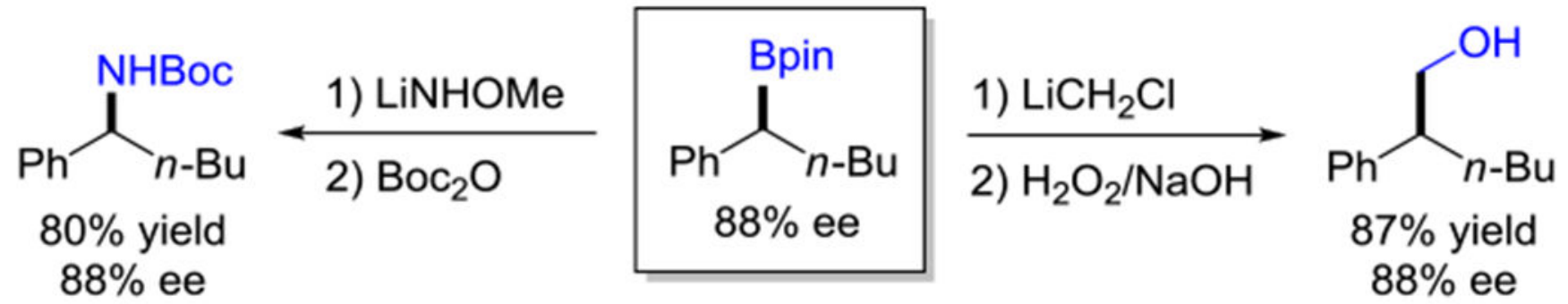<smiles>O=C(PC(c1ccccc1)c1ccncc1F)c1ccccc1</smiles>

$72 \%$ yield $88 \%$ ee

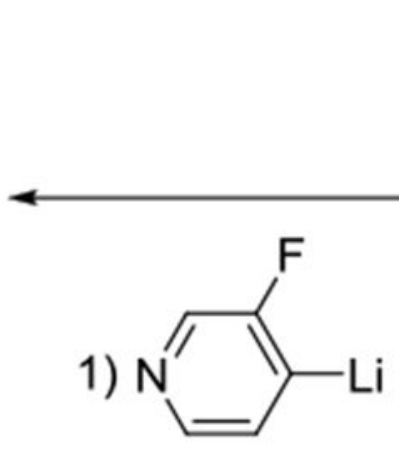

2) $\mathrm{TrocCl}$

3) $\mathrm{H}_{2} \mathrm{O}_{2} / \mathrm{NaOH}$

Figure 3.

Representative transformations of an enantioenriched benzylic boronic ester.

1)<smiles>[AlH2]c1cccs1</smiles>

2) NBS<smiles>CCCCC(c1ccccc1)c1cccs1</smiles>

$83 \%$ yield $88 \%$ ee 
Table 1.

Enantioconvergent borylation of a racemic benzylic chloride: effect of reaction parameters. ${ }^{\text {[a] }}$

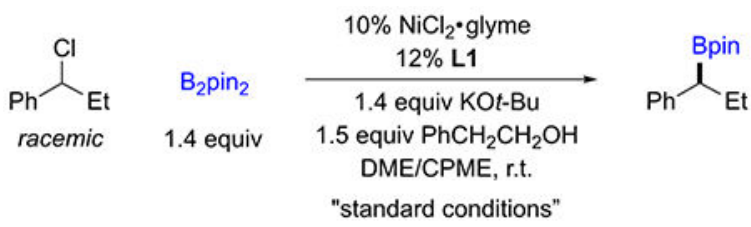

\begin{tabular}{|c|c|c|c|}
\hline entry & variation from the "standard conditions" & yield $(\%)^{[b]}$ & ee $(\%)^{[c]}$ \\
\hline 1 & none & 87 & 85 \\
\hline 2 & no $\mathrm{NiCl}_{2} \cdot$ glyme & $<2$ & - \\
\hline 3 & no $\mathbf{L 1}$ & 16 & - \\
\hline 4 & no $\mathrm{PhCH}_{2} \mathrm{CH}_{2} \mathrm{OH}$ & 24 & 73 \\
\hline 5 & $\mathbf{L} 2$, instead of $\mathbf{L} 1$ & 95 & $<2$ \\
\hline 6 & $\mathbf{L 3}$, instead of $\mathbf{L 1}$ & 98 & -40 \\
\hline 7 & $\mathbf{L 4}$, instead of $\mathbf{L 1}$ & 17 & -6 \\
\hline 8 & $0^{\circ} \mathrm{C}$, instead of r.t. & 52 & 84 \\
\hline 9 & DME only & 80 & 84 \\
\hline 10 & CPME only & 61 & 84 \\
\hline 11 & $5 \% \mathrm{NiCl}_{2} \cdot$ glyme, $6 \% \mathbf{L 1}$ & 78 & 80 \\
\hline 12 & 1.1 equiv $\mathrm{B}_{2} \operatorname{pin}_{2}, 1.1$ equiv $\mathrm{KO} t$ - $\mathrm{Bu}, 1.2$ equiv $\mathrm{PhCH}_{2} \mathrm{CH}_{2} \mathrm{OH}$ & 79 & 84 \\
\hline 13 & 1.0 equiv $\mathrm{H}_{2} \mathrm{O}$ & 58 & 79 \\
\hline 14 & under air in a closed vial & 47 & 80 \\
\hline
\end{tabular}

${ }^{[a]}$ All data are the average of two experiments.

${ }^{[b]}$ The yield was determined through GC analysis with $n$-dodecane as an internal standard.

${ }^{[c]}$ A negative value for ee signifies that the major product is the $\mathrm{R}$ enantiomer.

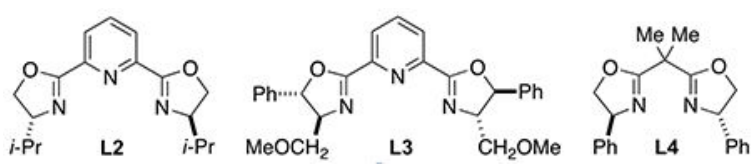


Table 2.

Enantioconvergent borylations of racemic benzylic chlorides: scope. ${ }^{\text {[a] }}$

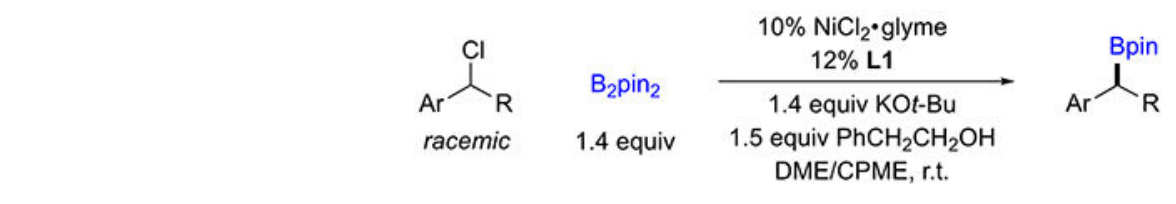

\begin{tabular}{lllcr}
\hline entry & Ar & R & yield (\%) $[\boldsymbol{b}]$ & ee (\%) \\
\hline 1 & $\mathrm{Ph}$ & $\mathrm{Et}$ & 81 & 86 \\
2 & $\mathrm{Ph}$ & $\mathrm{Me}$ & 64 & 82 \\
3 & $\mathrm{Ph}$ & $n-\mathrm{Bu}$ & 80 & 88
\end{tabular}

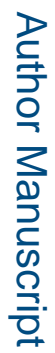

${ }_{4}{ }_{\mathrm{Ph}} \quad \mathrm{CH}_{2} \longrightarrow \mathrm{Me}^{\mathrm{Me}}{ }^{82}{ }_{88}$

\begin{tabular}{|c|c|c|c|c|}
\hline 5 & $\mathrm{Ph}$ & Сy & 61 & 78 \\
\hline 6 & $\mathrm{Ph}$ & $\mathrm{Bn}$ & 79 & 83 \\
\hline 7 & $\mathrm{Ph}$ & $\left(\mathrm{CH}_{2}\right)_{5}-\mathrm{OBn}$ & 66 & 80 \\
\hline 8 & $\mathrm{Ph}$ & $\left(\mathrm{CH}_{2}\right)_{5}-\mathrm{OBz}$ & 74 & 83 \\
\hline 9 & $\mathrm{Ph}$ & $\left(\mathrm{CH}_{2}\right)_{5}-\mathrm{Cl}$ & 82 & 86 \\
\hline 10 & $p$-tolyl & Et & 64 & 82 \\
\hline 11 & $m$-tolyl & Et & 77 & 82 \\
\hline 12 & $m$-anisyl & Et & 69 & 80 \\
\hline 13 & $o$-tolyl & Et & 88 & 86 \\
\hline 14 & $o$-fluorophenyl & Et & 85 & 81 \\
\hline 15 & 1-naphthyl & Et & 90 & 83 \\
\hline 16 & & Et & 86 & 51 \\
\hline 17 & & & 81 & 81 \\
\hline
\end{tabular}

${ }^{[a]}$ All data are the average of two experiments.

${ }^{[b]}$ Yield of purified product. 